\title{
Efecto de la distribución del tiempo de servicio de los usuarios primarios en las estadísticas de los espacios en blanco de un sistema de radio cognoscitivo
}

\author{
Effect of the service time distribution of the primary users in the statistics of a \\ cognitive radio system white spaces
}

HERNÁNDEZ-VALDEZ, Genaro†*, CRUZ-PÉREZ, Felipe Alejandro, LABASTIDA-CUADRA, José Omar y PÉREZ-SANCHEZ, Grethell Georgina

Universidad Autónoma Metropolitana - Unidad Azcapotzalco

ID 1er Autor: Genaro, Hernández-Valdez, ORC ID: 0000-0002-1280-3527, CVU CONACYT ID: 35223

ID $1^{\mathrm{er}}$ Coautor: Felipe Alejandro, Cruz-Pérez / ORC ID: 0000-0001-8328-3760, CVU CONACYTD ID: 20709

ID $2^{\text {do }}$ Coautor: José Omar, Labastida-Cuadra / ORC ID: 0000-0003-1067-8053, Researcher ID Thomson: G-3771-2019, CVU CONACYT ID: 973225

ID $3^{\text {er }}$ Coautor: Grethell Georgina, Pérez-Sanchez / ORC ID: 0000-0002-5505-6226, Researcher ID Thomson: C-86412018, CVU CONACYT ID: 230934

DOI: $10.35429 / J U S D .2019 .16 .5 .15 .23$

Recibido: 30 de Junio, 2019; Aceptado 30 de Septiembre, 2019

Resumen

La tecnología de radio cognoscitivo fue desarrollada para mejorar la eficiencia espectral en redes de comunicación móvil. Esto se logra al permitir que usuarios secundarios utilicen de manera oportunista y transparente los espacios en blanco de la red primaria. En este artículo se analiza el efecto de la distribución del tiempo de servicio de los usuarios primarios en las estadísticas de los espacios en blanco. En particular, se determinan los dos primeros momentos estandarizados de la duración de los espacios en blanco y del tiempo entre arribos de espacios en blanco, considerando las siguientes distribuciones para el tiempo de servicio de los usuarios primarios: log-normal, Weibull y Pareto. Un resultado relevante es que, para escenarios de baja (moderada o alta) carga de tráfico, la distribución exponencial (hiper-exponencial) es una opción razonable para modelar la duración de los espacios en blanco. Al caracterizar esta variable temporal, es posible utilizar el modelo de encendidoapagado para capturar la actividad de los canales primarios $y$, de esta forma, evaluar el desempeño de redes de radio cognoscitivo. Los resultados se obtuvieron utilizando técnicas de simulación de eventos discretos.

Estadísticas de los espacios en blanco

\begin{abstract}
Cognitive radio technology was developed for spectral efficiency improvement in mobile communication networks. This is achieved by allowing secondary users to opportunistically and transparently use the white spaces of the primary network. In this paper, the effect of the service time distribution of the primary users in the statistics of the white spaces is analyzed. In particular, the first two standardized moments of both, white space time and white space interarrival time are found, considering the following distributions for the primary service time: log-normal, Weibull, and Pareto. One of the most relevant results is that, for low (moderate or high) traffic load, the exponential (hiper-exponential) distribution is an excellent option for modelling the white space duration. Characterizing these variables allow us to use the on-off paradigm to capture the primary channel activity and, in this way, evaluate the performance of cognitive radio networks. Results were obtained by using discrete event simulation techniques.
\end{abstract}

Citación: HERNÁNDEZ-VALDEZ, Genaro, CRUZ-PÉREZ, Felipe Alejandro, LABASTIDA-CUADRA, José Omar y PÉREZ-SANCHEZ, Grethell Georgina. Efecto de la distribución del tiempo de servicio de los usuarios primarios en las estadísticas de los espacios en blanco de un sistema de radio cognoscitivo. Revista del Desarrollo Urbano y Sustentable. 2019. 5-16: $15-23$

\footnotetext{
* Correspondencia del Autor (ghv.uam@gmail.com)

$\dagger$ Investigador contribuyendo como primer autor
} 


\section{Introducción}

Un sistema de radio cognoscitivo se compone de dos redes (red primaria y red secundaria) que comparten una misma banda de frecuencia para proporcionar servicios de comunicación a sus abonados, inclusive, éstas redes pueden operar en la misma área geográfica, ver figura 1 . La red primaria tiene la licencia de la banda de frecuencia y, por lo tanto, tiene prioridad para usarla; a los usuarios de esta red se les conoce como usuarios primarios (UP).

La red secundaria usa de forma oportunista (y transparente para la red primaria) los recursos de radio (canales) de la red primaria; a los usuarios de esta red se les conoce como usuarios secundarios (US). De esta forma, cuando arriba un US al sistema, se le asigna un canal que no esté siendo usado por la red primaria (a estos canales se les conoce como espacios en blanco o huecos espectrales). Si durante la sesión de un US, la red primaria requiere el canal que está usando, la red secundaria libera inmediatamente dicho canal y busca algún otro espacio en blanco para darle continuidad a la sesión del US (a éste proceso se le conoce como transferencia espectral). De no encontrar un espacio en blanco, la sesión del US tendrá una terminación abrupta o se formará en una cola de espera, esto dependerá del tipo de servicio que curse el US y de la política de manejo de recursos de la red secundaria.

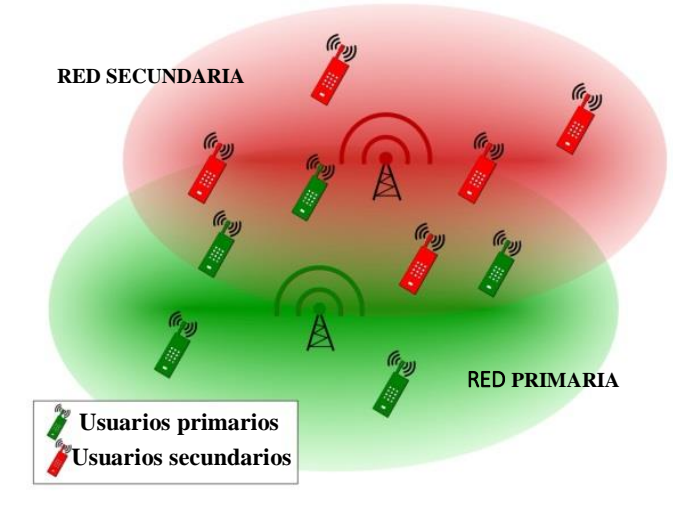

Figura 1 Diagrama conceptual de un sistema de radio cognoscitivo

Fuente: Elaboración Propia

Debido a la naturaleza aleatoria tanto de los arribos de peticiones de servicio como del tiempo durante el cual se usan los canales en la red primaria, no existe certidumbre del tiempo durante el cual la red secundaria puede disponer de los espacios en blanco con los que cuenta en un momento dado.
Basados en mediciones recolectadas en sistemas reales, se han propuesto diferentes funciones de distribución de probabilidades (pdf) para modelar la duración de los espacios en blanco. De esta forma, se han desarrollado modelos matemáticos para estudiar el desempeño de redes de radio cognoscitivo considerando un modelo encendido-apagado para representar la actividad de los canales de la red primaria.

En este modelo, el tiempo de encendido de un canal está representado por el tiempo de servicio de los usuarios primarios y el tiempo de apagado está representado por la duración de los espacios en blanco, este modelo se muestra en la figura 2. Sin embargo, este modelo requiere capturar la actividad individual de cada uno de los canales de la red primaria, por lo que su análisis matemático y computacional puede ser complejo. Además, para que el modelo de encendido-apagado capture de forma adecuada el comportamiento de los espacios en blanco, se requiere determinar la relación entre las estadísticas de los espacios en blanco y la distribución del tiempo de servicio y el proceso de arribos de los usuarios primarios. Hasta donde tenemos conocimiento, no existe un estudio en este sentido. El presente trabajo representa un punto de inicio en esta dirección de investigación. En particular, en este trabajo se investiga el impacto de la distribución del tiempo de servicio en las estadíticas de los espacios en blanco.

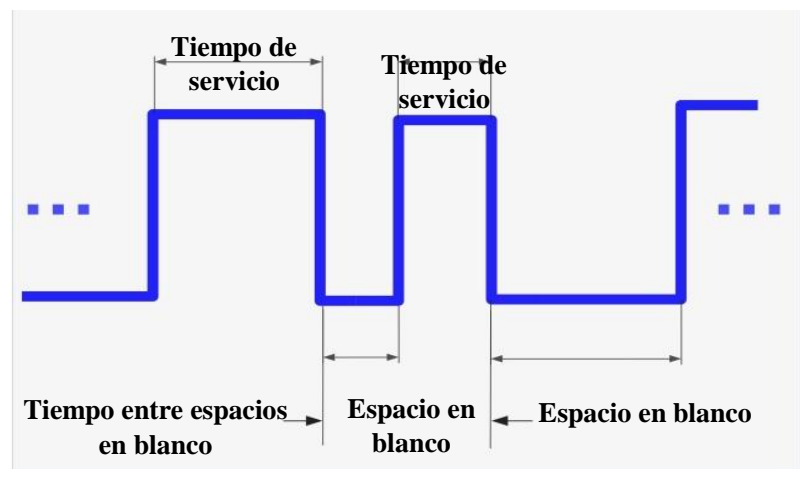

Figura 2 Modelo encendido-apagado que describe la actividad de los canales primaries

Fuente: Elaboración Propia

El tiempo de servicio, tradicionalmente y por simplicidad matemática, se modelaba como una variable aleatoria exponencialmente distribuida. Sin embargo, diversos estudios han mostrado que los tiempos de servicio de aplicaciones actuales siguen distribuciones de cola pesada como la log-normal, Pareto, y Weibull.

HERNÁNDEZ-VALDEZ, Genaro, CRUZ-PÉREZ, Felipe Alejandro, LABASTIDA-CUADRA, José Omar y PÉREZ-SANCHEZ, Grethell Georgina. Efecto de la distribución del tiempo de servicio de los usuarios primarios en las estadísticas de los espacios en blanco de un sistema de radio cognoscitivo. Revista del Desarrollo Urbano y Sustentable. 2019. 
Es por esto que, en este trabajo, usamos estas distribuciones para modelar el tiempo de servicio de los UP.

\section{Objetivo}

El objetivo principal de este trabajo es determinar las estadísticas de los espacios en blanco (valor esperado y coeficiente de variación) considerando que la distribución del tiempo de servicio de los UP sigue una distribución de cola pesada (Weibull, Pareto o log-normal) y el proceso de arribos de los UP es de Poisson.

\section{Metodología}

En este trabajo se desarrolla un método de simulación de eventos discretos para obtener las estadísticas (valor medio y coeficiente de variación) y el histograma normalizado tanto de la duración de los espacios en blanco como del tiempo entre arribos de espacios en blanco. Para esto, el modelo de simulación captura aspectos relevantes del sistema tales como el tiempo entre arribos de peticiones de servicio de usuarios primarios, tiempo que un usuario primario permanece dentro del sistema, tráfico ofrecido y probabilidad de bloqueo requerida en la red primaria. Los modelos matemáticos de las variables temporales involucradas en la operación del sistema y la construcción del modelo de simulación de eventos discretos se presentan con mayor detalle en la sección titulada "Modelo del sistema y simulación" del presente artículo.

\section{Contribución de la investigación}

La contribución principal de este trabajo es el desarrollo de un modelo basado en simulación de eventos discretos que permite investigar el efecto del tipo de distribución y del valor de los dos primeros momentos centralizados del tiempo de servicio de los usuarios primarios en las estadísticas de los espacios en blanco, considerando diferentes condiciones de carga ofrecida al sistema primario. Con base en los resultados obtenidos es posible proponer distribuciones tipo fase para modelar la duración de los espacios en blanco y de está manera desarrollar modelos de teletráfico matemáticamente tratables que permitan analizar el desempeño de redes de radio cognoscitivo.
El resto del artículo está organizado como se indica a continuación. Primeramente, en la sección "Modelo del sistema y simulación" se describe el modelo del sistema, las variables aleatorias involucradas y el simulador de eventos discretos desarrollado para analizar el sistema. Después de esto, en la sección "Resultados" se presentan y analizan resultados numéricos relevantes. Finalmente, en la sección "Conclusiones" se presentan las conclusiones del artículo y el trabajo a futuro.

\section{Modelo del sistema y simulación}

Para estudiar el efecto del tiempo de servicio de los UP en las estadísticas de los espacios en blanco, se considera una red primaria, la cual cuenta con un número total de $S$ canales. La política de admisión de llamada considera que las peticiones de servicio que encuentran todos los canales ocupados son bloqueadas. Una sesión que es admitida en la red primaria abandona el sistema hasta que termina su sesión.

\section{Parámetros del sistema}

Se considera que el proceso de arribos de la red primaria es de Poisson, por lo tanto, el tiempo entre arribos de nuevas sesiones es una variable aleatoria $\mathbf{X}_{a}$ con distribución exponencial negativa con tasa $\lambda=1 / E\left\{\mathbf{X}_{a}\right\}$ arribos/s, donde $E\left\{\mathbf{X}_{a}\right\}$ es el valor esperado (media) del tiempo entre arribos. Se considera que el tiempo de servicio de las sesiones primarias es una variable aleatoria $\mathbf{X}_{\boldsymbol{s}}$ con distribución de cola pesada con valor medio $E\left\{\mathbf{X}_{s}\right\}=180$ s y coeficiente de variación $\operatorname{CoV}\left\{\mathbf{X}_{s}\right\}$. En particular, se consideran tres tipos de distribuciones diferentes para modelar el tiempo de servicio de los usuarios primarios: lognormal, Pareto y Weibull. En la tabla 1 se muestran las expresiones matemáticas tanto de la pdf como del valor medio $E\left\{\mathbf{X}_{s}\right\}$ y de la varianza $V\left\{\mathbf{X}_{s}\right\}$ de estas distribuciones. En este trabajo, se considera, sin pérdida de generalidad, que el valor medio del tiempo de servicio es $E\left\{\mathbf{X}_{s}\right\}=180 \mathrm{~s}$ y se consideran tres valores diferentes del coeficiente de variación del tiempo de servicio: $\operatorname{CoV}\left\{\mathbf{X}_{s}\right\}=\{2,5$ y 10$\}$. En la tabla 2, se muestran los valores de los parámetros de escala, forma y locación de estas distribuciones, con los que se obtienen los valores deseados de media y coeficiente de variación del tiempo de servicio de los UP. 
El tráfico ofrecido en la red primaria está dado por $a=\lambda \cdot E\left\{\mathbf{X}_{s}\right\}$. En esta red, la probabilidad de bloqueo $\left(P_{b}\right)$ está dada por la fórmula Erlang $B$ :

$$
P_{b}=B(S, a)=\frac{a^{S} / S !}{\sum_{j=0}^{S} a^{j} / j !}
$$

\begin{tabular}{|c|c|c|}
\hline \multicolumn{2}{|c|}{ Distribución / pdf } & Parámetros/media/varianza \\
\hline \multirow{3}{*}{ है } & \multirow{3}{*}{$\frac{e^{\left(\frac{-\left[\log \left(\frac{x}{m}\right)\right]^{2}}{2 \sigma^{2}}\right)}}{x \sigma \sqrt{2 \pi}}$} & Escala: $\mathrm{m}>0$, Forma: $\sigma>0$ \\
\hline & & $E\left\{\mathbf{X}_{s}\right\}=m e^{\frac{\sigma^{2}}{2}}$ \\
\hline & & $V\left\{\mathbf{X}_{s}\right\}=m^{2} e^{\sigma^{2}}\left(e^{\sigma^{2}}-1\right)$ \\
\hline \multirow{3}{*}{ 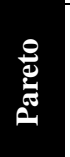 } & \multirow{3}{*}{$\frac{c a^{c}}{x^{c+1}}$} & Localidad: a > 0, Forma: $\mathbf{c}>0$ \\
\hline & & $E\left\{\mathbf{X}_{s}\right\}=\frac{c a}{(c-1)}, c>1$ \\
\hline & & $V\left\{\mathbf{X}_{s}\right\}=\frac{c a^{2}}{\left[(c-1)^{2}(c-2)\right]}, c>2$ \\
\hline \multirow{3}{*}{$\begin{array}{l}\overline{\bar{B}} \\
\frac{\overline{0}}{\bar{d}} \\
3\end{array}$} & \multirow{3}{*}{$\frac{\beta x^{\beta-1}}{\left(\eta e^{\left(\frac{x}{\eta}\right)}\right)^{\beta}}$} & Escala: $\eta>0$, Forma: $\beta>0$ \\
\hline & & $E\left\{\mathbf{X}_{s}\right\}=\eta \Gamma\left[\frac{\beta+1}{\beta}\right]$ \\
\hline & & $V\left\{\mathbf{X}_{s}\right\}=\eta^{2}\left(\Gamma\left[\frac{\beta+2}{\beta}\right]-\left\{\Gamma\left[\frac{\beta+1}{\beta}\right]\right\}^{2}\right)$ \\
\hline
\end{tabular}

Tabla 1 Funciones de distribución de probabilidades consideradas para modelar el tiempo de servicio de los usuarios primaries

Fuente: Elaboración Propia

\begin{tabular}{|c|c|c|c|}
\hline Distribución & $E\left\{\mathbf{X}_{s}\right\}$ & $\operatorname{CoV}\left\{\mathrm{X}_{s}\right\}$ & Parámetros \\
\hline \multirow{6}{*}{ Lognormal } & \multirow{6}{*}{180} & \multirow{2}{*}{2} & $m=80.49844718$ \\
\hline & & & $\sigma=1.26863624$ \\
\hline & & \multirow{2}{*}{5} & $m=35.30090432$ \\
\hline & & & $\sigma=1.80501981$ \\
\hline & & \multirow{2}{*}{10} & $m=17.91066942$ \\
\hline & & & $\sigma=2.14828315$ \\
\hline \multirow{6}{*}{ Pareto } & \multirow{6}{*}{180} & \multirow{2}{*}{2} & $a=95.0155281$ \\
\hline & & & $c=2.11803398$ \\
\hline & & \multirow{2}{*}{5} & $a=90.88243776$ \\
\hline & & & $c=2.0198039$ \\
\hline & & \multirow{2}{*}{10} & $a=90.22388198$ \\
\hline & & & $c=2.00498756$ \\
\hline \multirow{6}{*}{ Weibull } & \multirow{6}{*}{180} & \multirow{2}{*}{2} & $\eta=103.54491987$ \\
\hline & & & $\beta=0.54269256$ \\
\hline & & \multirow{2}{*}{5} & $\eta=22.84557338$ \\
\hline & & & $\beta=0.31134072$ \\
\hline & & \multirow{2}{*}{10} & $\eta=4.81669224$ \\
\hline & & & $\beta=0.23320675$ \\
\hline
\end{tabular}

Tabla 2 Valor de los parámetros de las distribuciones log-normal, Pareto y Weibull con los que se obtienen el valor esperado y coeficiente de variación deseados del tiempo de servicio de los usuarios primaries

Fuente: Elaboración Propia

El tráfico cursado $\left(a_{c}\right)$ en la red primaria se puede calcular de la siguiente forma:

$a_{c}=\left(1-P_{b}\right) a$

El factor de utilización ( $\rho$ ) de los canales primarios se define como sigue:

$\rho=\frac{a_{c}}{S}$
Para estudiar el efecto de la distribución del tiempo de servicio de los UP en las estadísticas de los espacios en blanco se desarrolla un modelo de simulación de eventos discretos.

\section{Modelo de simulación}

El simulador de eventos discretos para evaluar las estadísticas de los espacios en blanco tiene como parámetros de entrada los siguientes: número total de canales $S$; el tipo de distribución, valor medio y coeficiente de variación del tiempo de servicio de los UP; el factor de utilización $\rho$ de los canales primarios o el valor máximo permitido de la probabilidad de bloqueo $P_{\text {breq }}$ (sólo se debe proporcionar uno de estos dos parámetros, ya que al darle valor a uno de ellos el otro queda determinado). Los valores de los parámetros de forma y de localidad o escala se obtienen a partir del valor medio $E\left\{\mathbf{X}_{s}\right\}$ y coeficiente de variación $\operatorname{CoV}\left\{\mathbf{X}_{s}\right\}$ del tiempo de servicio de los UP; para esto se utilizan las expresiones indicadas en la tabla 1. Similarmente, se utilizan las expresiones (1)-(3) y los valores de $S$ y de $\rho$ (o de $\left.P_{\text {breq }}\right)$, para determinar el valor del tráfico ofrecido $a$ (y de la tasa de arribos $\lambda$ ) que se debe considerar. El simulador de eventos discretos tiene como objetivo obtener el valor medio y el coeficiente de variación tanto de la duración de los espacios en blanco $\mathbf{X}_{\boldsymbol{B}}$ como del tiempo entre arribos de espacios en blanco $\mathbf{X}_{\boldsymbol{A}}$. Básicamente, el simulador considera diversas variables aleatorias involucradas en la operación del sistema: tiempo entre arribos de peticiones de servicio de UP, duración del tiempo de servicio de sesiones de UP, duración de los espacios en blanco, tiempo entre arribos de espacios en blanco.

El estado del sistema (representado por el número de canales disponibles) puede cambiar debido a dos tipos de eventos: el evento "arribo" (una nueva petición de servicio llega al sistema) y el evento "abandono" (una sesión finaliza exitosamente su servicio). A cada evento se le asigna una etiqueta temporal, la cual indica el momento en el que ocurre dicho evento. Los eventos se insertan en una lista ordenada y se procesan de forma creciente de acuerdo al tiempo en el que ocurren. La duración de los espacios en blanco y los tiempos entre arribos de espacios en blanco se recolectan por canal. 
Los detalles del simulador se muestran en el diagrama de flujo de la figura 3.

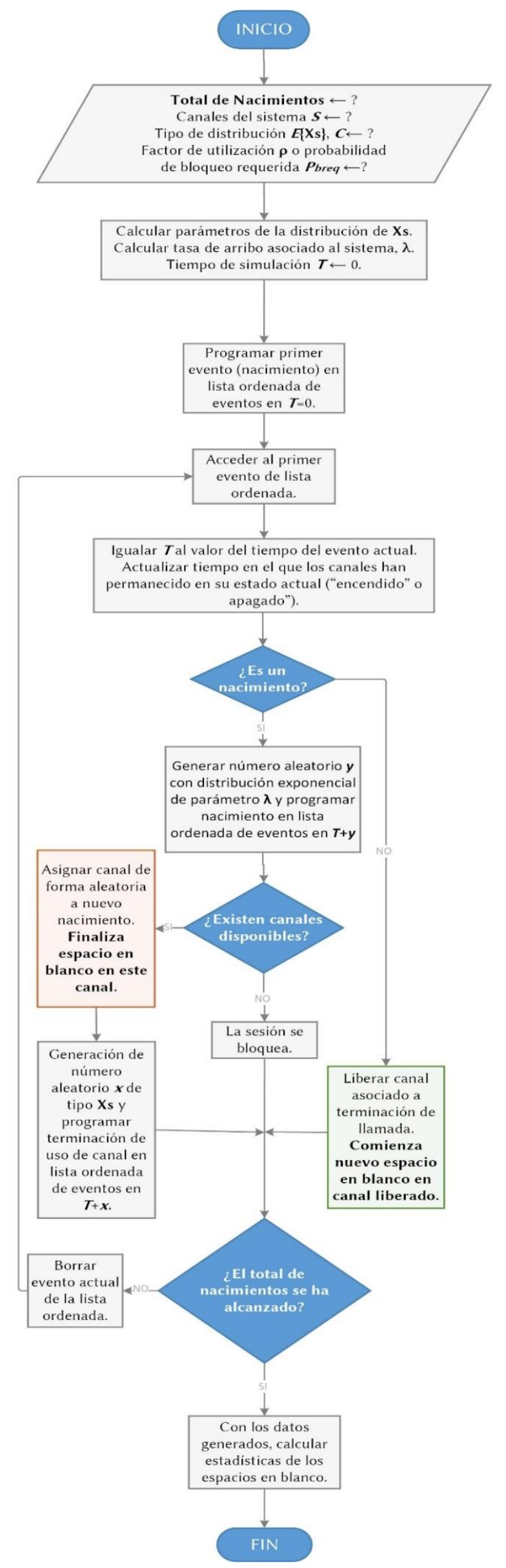

Figura 3 Operación general del simulador de eventos discretos

Fuente: Elaboración Propia

\section{Resultados}

Utilizando la metodología de análisis descrita en la sección anterior, en esta sección se presentan resultados numéricos para estudiar el efecto de la distribución del tiempo de servicio de los usuarios primarios en las estadísticas de la duración y del tiempo entre arribos de espacios en blanco. Estas estadísticas se obtienen para tres diferentes valores de la probabilidad de bloqueo: $0.5 \%$ (escenario de baja carga de tráfico), $5 \%$ (escenario de carga moderada de tráfico) y $50 \%$ (escenario de alta carga de tráfico).

En la práctica, los sistemas de comunicación móvil se diseñan para que la probabilidad de bloqueo se encuentre entre el 2 $\%$ y el $5 \%$ en condiciones moderadas de tráfico. En sistemas reales, los escenarios de alta carga de tráfico ocurren, por ejemplo, durante eventos de catástrofe o en días festivos, mientras que los escenarios de baja carga ocurren, por ejemplo, a altas horas de la noche o durante la madrugada.

En las tablas 3 y 4 se reporta el coeficiente de variación (renglones iluminados en color azul claro) y la media (renglones sin iluminar) de la duración de los espacios en blanco $\mathbf{X}_{\boldsymbol{B}}$ (tabla 3) y del tiempo entre arribos de los espacios en blanco $\mathbf{X}_{\boldsymbol{A}}$ (tabla 4). Estos resultados corresponden a un sistema primario con $S=30$ canales, valor medio del tiempo de servicio de los UP $E\left\{\mathbf{X}_{s}\right\}=180 \mathrm{~s}$. En estas tablas se reportan resultados para los tres escenarios de evaluación descritos en el párrafo anterior (es decir, probabilidades de bloqueo del $0.5 \%$, $5 \%$ y $50 \%$ ). En las tablas 3 y 4, el tipo de distribución y el coeficiente de variación del tiempo de servicio de los UP se consideran como parámetros.

De las tablas 3 y 4 se pueden extraer los siguientes resultados relevantes. La tabla 3 muestra que el valor del coeficiente de variación de la duración de los espacios en blanco se encuentra alrededor de 1.1, $1.2 \mathrm{y}$ entre 1.2 y 1.3, para escenarios de baja, moderada y alta carga de tráfico, respectivamente. Estos resultados sugieren que la duración de los espacios en blanco se puede modelar con una distribución exponencial negativa para escenarios de bajo tráfico o con una distribución hiper-exponencial de bajo orden (por ejemplo de orden 2 o 3) para esenarios de tráfico moderado o alto. 
El usar distribuciones tipo fase para la duración de los espacios en blanco, permite desarrollar modelos de teletráfico relativamente sencillos para analizar el desempeño de redes de radio cognoscitivo.

\begin{tabular}{|c|c|c|c|c|}
\hline \multirow{3}{*}{$\mid \begin{array}{l}n \\
0 \\
z \\
z \\
z \\
z\end{array}$} & \multirow{3}{*}{$\frac{\tilde{y}}{\tilde{y}}$} & \multicolumn{3}{|c|}{$\begin{array}{l}\text { Valor medio y coeficiente de variación de } \\
\text { los espacios en blanco }\left(E\left\{X_{B}\right\} / C o V\left\{X_{B}\right\}\right)\end{array}$} \\
\hline & & \multicolumn{3}{|c|}{ Probabilidad de bloqueo $P_{b}$} \\
\hline & & $0.5 \%$ & $5 \%$ & $50 \%$ \\
\hline \multirow{6}{*}{ 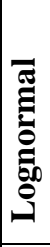 } & \multirow[t]{2}{*}{2} & 104.63 & 49.542 & 5.8324 \\
\hline & & 1.0940 & 1.2034 & 1.2447 \\
\hline & \multirow[t]{2}{*}{5} & 106.16 & 49.213 & 5.8384 \\
\hline & & 1.0997 & 1.2175 & 1.2628 \\
\hline & \multirow[t]{2}{*}{10} & 103.66 & 50.026 & 5.9836 \\
\hline & & 1.1076 & 1.2298 & 1.2734 \\
\hline \multirow{6}{*}{ } & \multirow[t]{2}{*}{2} & 104.72 & 49.330 & 5.8501 \\
\hline & & 1.0792 & 1.1888 & 1.2389 \\
\hline & \multirow[t]{2}{*}{5} & 105.39 & 49.144 & 5.8638 \\
\hline & & 1.0767 & 1.1846 & 1.2376 \\
\hline & \multirow[t]{2}{*}{10} & 105.09 & 49.332 & 5.8753 \\
\hline & & 1.0778 & 1.1891 & 1.2381 \\
\hline \multirow{6}{*}{\begin{tabular}{|l}
$\overline{\bar{z}}$ \\
$\overline{\overline{0}}$ \\
$\bar{z}$
\end{tabular}} & \multirow[t]{2}{*}{2} & 105.55 & 48.997 & 5.8743 \\
\hline & & 1.0979 & 1.217 & 1.2587 \\
\hline & \multirow[t]{2}{*}{5} & 106.38 & 48.788 & 5.8178 \\
\hline & & 1.1082 & 1.2431 & 1.2810 \\
\hline & \multirow[t]{2}{*}{10} & 107.88 & 48.807 & 5.8351 \\
\hline & & 1.1162 & 1.2378 & 1.3197 \\
\hline
\end{tabular}

Tabla 3 Valor medio y coeficiente de variación de la duración de espacios en blanco para $S=30, E\left\{\mathbf{X}_{s}\right\}=180$, con el $\operatorname{CoV}\left\{\mathbf{X}_{s}\right\}$ y $P_{b}$ cómo parámetros

Fuente: Elaboración Propia

\begin{tabular}{|c|c|c|c|c|}
\hline \multirow{3}{*}{$\begin{array}{l}x \\
0 \\
0 \\
\frac{x}{z} \\
z\end{array}$} & \multirow{3}{*}{$\frac{\pi}{2}$} & \multicolumn{3}{|c|}{$\begin{array}{c}\text { Valor medio y coeficiente de variación } \\
\text { del tiempo entre arribos de espacios en } \\
\text { blanco }\left(E\left\{X_{\mathrm{A}}\right\} / \operatorname{CoV}\left\{\mathrm{X}_{\mathrm{A}}\right\}\right)\end{array}$} \\
\hline & & \multicolumn{3}{|c|}{ Probabilidad de bloqueo $P_{b}$} \\
\hline & & $0.5 \%$ & $5 \%$ & $50 \%$ \\
\hline \multirow{6}{*}{ 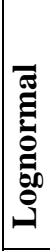 } & \multirow[t]{2}{*}{2} & 285.23 & 229.02 & 186.01 \\
\hline & & 1.3427 & 1.5809 & 1.9416 \\
\hline & \multirow[t]{2}{*}{5} & 284.85 & 228.97 & 186.63 \\
\hline & & 2.8609 & 3.6695 & 4.5217 \\
\hline & \multirow[t]{2}{*}{10} & 285.46 & 228.54 & 182.74 \\
\hline & & 5.9036 & 6.2688 & 7.3991 \\
\hline \multirow{6}{*}{ } & \multirow[t]{2}{*}{2} & 285.29 & 229.43 & 186.31 \\
\hline & & 1.0977 & 1.2968 & 1.3642 \\
\hline & \multirow[t]{2}{*}{5} & 285.15 & 228.83 & 185.67 \\
\hline & & 1.1312 & 1.1665 & 1.3127 \\
\hline & \multirow[t]{2}{*}{10} & 285.06 & 228.86 & 185.08 \\
\hline & & 1.1057 & 1.2881 & 1.3579 \\
\hline \multirow{6}{*}{$\begin{array}{l}\overline{\bar{J}} \\
\overline{0} \\
\overline{0} \\
\overline{3}\end{array}$} & \multirow[t]{2}{*}{2} & 285.67 & 229.22 & 185.93 \\
\hline & & 1.3242 & 1.5969 & 1.9455 \\
\hline & \multirow[t]{2}{*}{5} & 285.14 & 230.28 & 186.06 \\
\hline & & 3.1221 & 4.1699 & 4.9083 \\
\hline & \multirow[t]{2}{*}{10} & 285.27 & 229.44 & 186.68 \\
\hline & & 3.9757 & 5.9494 & 11.718 \\
\hline
\end{tabular}

Tabla 4 Valor medio y coeficiente de variación del tiempo entre arribos de espacios en blanco para $S=30$, $E\left\{\mathbf{X}_{s}\right\}=180$, con el $\operatorname{CoV}\left\{\mathbf{X}_{s}\right\}$ y $P_{b}$ cómo parámetros. Fuente: Elaboración Propia
Es importante notar que, en escenarios donde la probabilidad de bloqueo es mucho menor a $0.5 \%$ (por ejemplo, para $P_{b}=1 \times 10^{-6}$ ), practicamente todo el tráfico ofrecido se cursa (es decir, $\left.a_{c} \approx a\right)$. Por lo tanto, en estos escenarios, debido a la propiedad "sin memoria" de la distribución exponencial negativa (la cual se utiliza para modelar el tiempo entre arribos de los UP) y a la insensibilidad al tipo de distribución del tiempo de servicio de un sistema de "llamada bloqueada/llamada perdida", la distribución de la duración de los espacios en blanco se puede aproximar con gran exactitud con una distribución exponencial negativa con valor medio dado por $\left[\left(S \cdot E\left\{\mathbf{X}_{s}\right\} / a\right)-E\left\{\mathbf{X}_{s}\right\}\right]$, donde el primer término de ésta expresión representa el tiempo medio entre arribos por canal cuando ninguna petición de servicio se bloquea $\left(a_{c}=a\right)$.

Por ejemplo, para una $P_{b}=1 \times 10^{-6}, S=30$ y $E\left\{\mathbf{X}_{s}\right\}=180 \mathrm{~s}$, se utiliza (1) para obtener el tráfico ofrecido $a=10.945$ Erlangs, por lo tanto el valor medio de la duración de espacios en blanco es $E\left\{\mathbf{X}_{\boldsymbol{B}}\right\} \approx 313.38$ s. El valor que se obtiene para $E\left\{\mathbf{X}_{\boldsymbol{B}}\right\}$ utilizando el simulador desarrollado en la sección anterior fue de $310 \mathrm{~s}$. Por supuesto, la diferencia entre estos dos valores disminuye conforme se consideran probabilidades de bloqueo cada vez más pequeñas. Observe que esta situación no ocurre para el tiempo entre arribos de espacios en blanco debido a que, de acuerdo a la figura 2 , este tiempo es igual a la suma del tiempo de servicio y de la duración del espacio en blanco, es decir, $\mathbf{X}_{\boldsymbol{A}}=\mathbf{X} \boldsymbol{s}+\mathbf{X}_{\boldsymbol{B}}$. Lo que si es interesante observar es que, para probabilidades de bloqueo mucho menores a $0.5 \%, E\left\{\mathbf{X}_{\boldsymbol{A}}\right\} \approx S \cdot E\left\{\mathbf{X}_{s}\right\} / a$.

La tabla 4 muestra que el coeficiente de variación del tiempo entre arribos de espacios en blanco es considerablemente mayor a $1 \mathrm{y}$ aumenta conforme el coeficiente de variacion del tiempo de servicio de los UP aumenta, esto es particularmente cierto para las distribuciones log-normal y Weibull; para la distribución Pareto éste párametro varia entre 1.09 y 1.37. En particular, para los escenarios de baja, moderada y alta carga de tráfico, $\operatorname{CoV}\left\{\mathbf{X}_{A}\right\}$ llega a ser tan grande como 5.90, 6.26 y 11.7 , respectivamente. 
Finalmente, en los Gráficos 1-3 (4-6) se presenta el histograma normalizado de la duración de los espacios en blanco (del tiempo entre arribos de espacios en blanco) para el escenario de alta carga de tráfico, es decir, $P_{b}=50 \%$, considerando que el tiempo de servicio de los usuarios primarios es logNormal, Pareto y Weibull, respectivamente. En estos Gráficos se utilizaron los siguientes parámetros: $S=30, E\left\{\mathbf{X}_{s}\right\}=180, \operatorname{CoV}\left\{\mathbf{X}_{s}\right\}=10$.

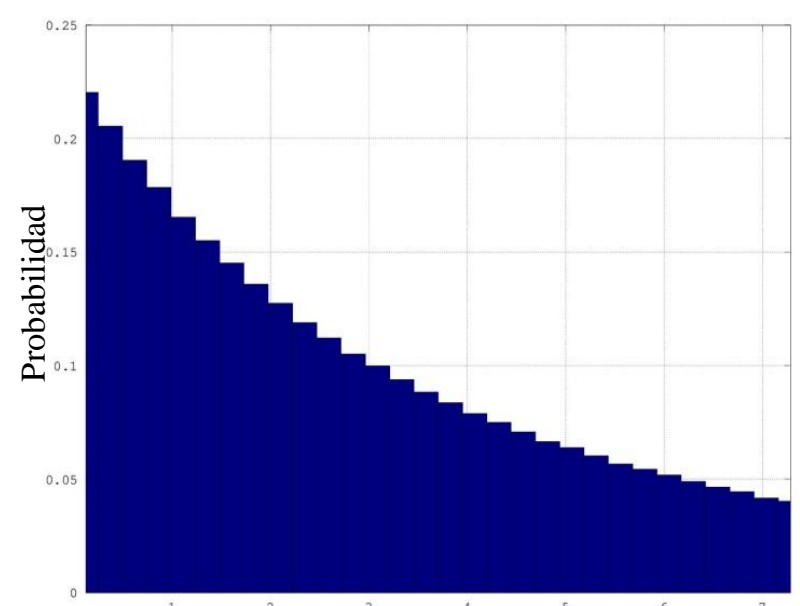

Histograma normalizado

Gráfico 1 Histograma normalizado de la duración de espacios en blanco, considerando tiempo de servicio de los UP log-normal

Fuente: Elaboración Propia

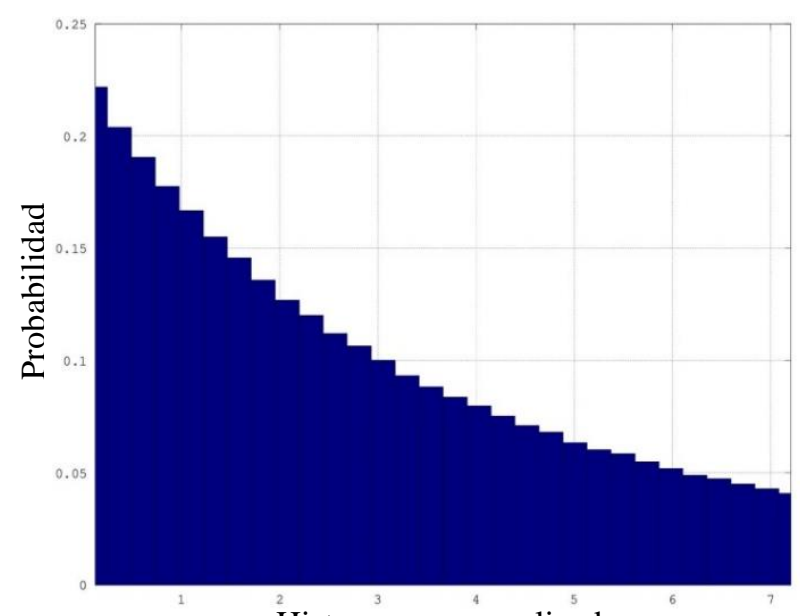

Histograma normalizado

Gráfico 2 Histograma normalizado de la duración de espacios en blanco, considerando tiempo de servicio de los UP Pareto

Fuente: Elaboración Propia

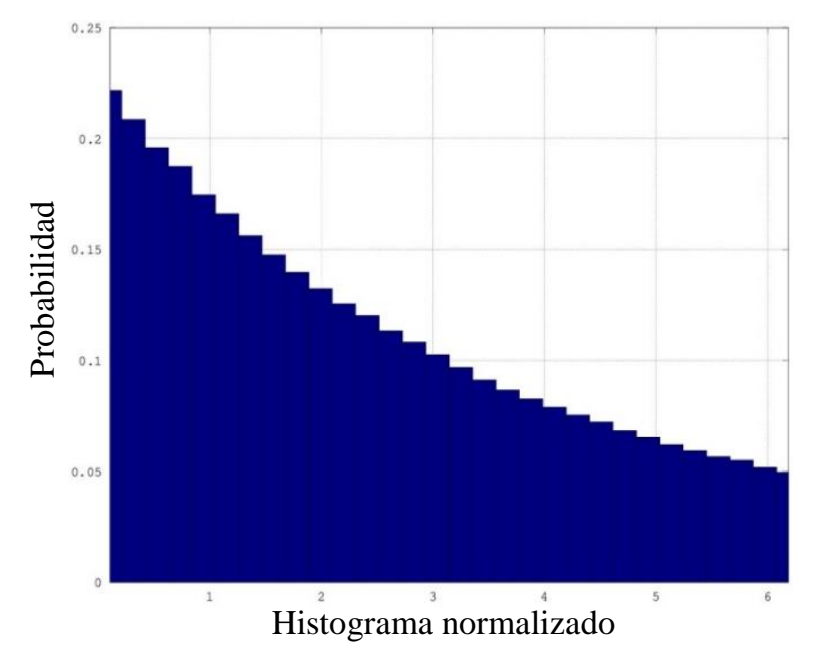

Gráfico 3 Histograma normalizado de la duración de espacios en blanco, considerando tiempo de servicio de los UP Weibull

Fuente: Elaboración Propia

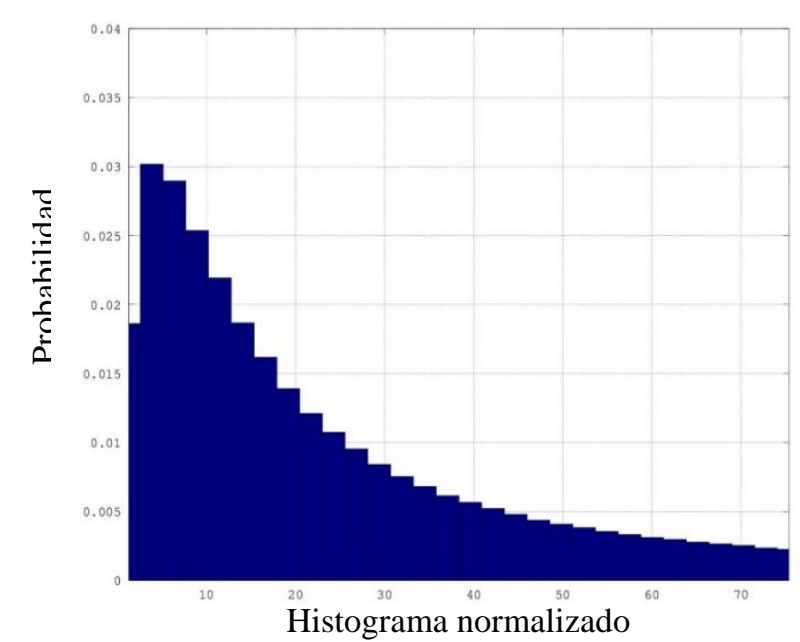

Gráfico 4 Histograma normalizado del tiempo entre arribos de espacios en blanco, considerando tiempo de servicio de los UP log-normal

Fuente: Elaboración Propia

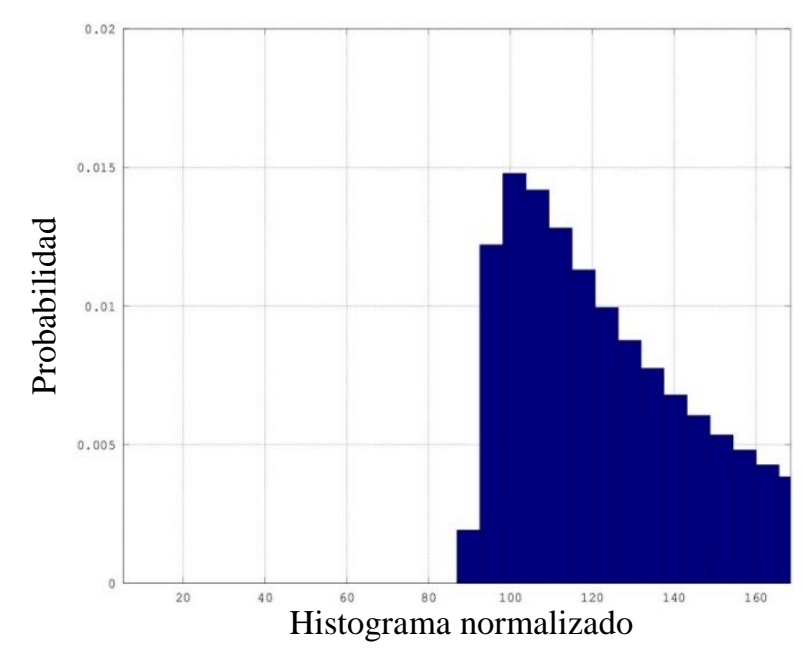

Gráfico 5 Histograma normalizado del tiempo entre arribos de espacios en blanco, considerando tiempo de servicio de los UP Pareto

Fuente: Elaboración Propia 


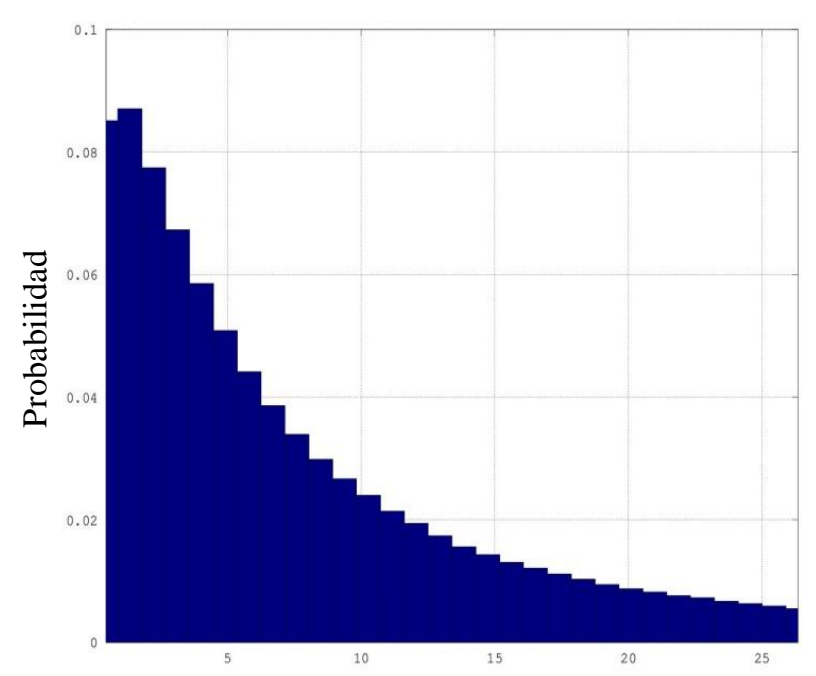

Histograma

Gráfico 6 Histograma normalizado del tiempo entre arribos de espacios en blanco, considerando tiempo de servicio de los UP Weibull

Fuente: Elaboración Propia

\section{Conclusiones}

En este trabajo se estudió el efecto del tipo de distribución y del valor de los parámetros del tiempo de servicio de los usuarios primarios en las estadísticas de los espacios en blanco de una red de radio cognoscitivo considerando que el proceso de arribos de la red primaria es de Poisson. Se encontró que para escenarios de muy baja carga de tráfico, el coeficiente de variación de la duración de los espacios en blanco es muy cercano a $1 \mathrm{y}$, por lo tanto, la distribución exponencial negativa representa un modelo adecuado para esta variable temporal.

Para escenarios de tráfico moderado y alto tráfico el coeficiente de variación se encontró entre 1.18 y 1.32 , por lo que el modelo hiper-exponencial es una alternativa para modelar esta variable temporal. El utilizar distribuciones tipo fase para modelar la duración de los espacios en blanco nos permite desarrollar modelos matématicos basados en la teoría de colas para análizar el desempeño de sistemas de radio cognoscitivo. Esto último representa un tema de investigación de nuestro trabajo futuro.

También, como trabajo futuro se pretende estudiar el efecto del proceso de arribos de los usuarios primarios en las estadísticas de los espacios en blanco.

\section{Agradecimientos}

Agradecemos el apoyo financiero de la Universidad Autónoma Metropolitana, del Centro de Investigación y de Estudios Avanzados del IPN y del Programa para el Desarrollo Profesional Docente (PRODEP) de la SEP, para la realización del presente trabajo. También agradecemos a la Dra. Sandra Lirio Castellanos López por su apoyo en el análisis de resultados y en la construcción del simulador desarrollado en este trabajo. De igual forma, agradecemos al Ing. Víctor Hugo Caro Martínez por su apoyo en la edición de figuras y tablas, en la construcción del simulador y en la generación de resultados numéricos de este trabajo.

\section{Referencias}

Asmussen S., Nerman O. and Olson M. (1996). Fitting Phase-Type Distributions via the EM Algorithm. Scandinavian Journal of Statistics, 23(4), 419-441.

Castellanos-López S. L., Cruz-Pérez F. A., and Hernandez-Valdez G. (2011). Performance of Cognitive Radio Networks under ON/OFF and Poisson Primary Arrival Models. IEEE International Symposium on Personal, Indoor and Mobile Radio Communications. 609-613.

Castellanos-López S. L., Cruz-Pérez F.A., Rivero Ángeles M. E., and Hernandez-Valdez G. (2013). Performance Comparison of VoIP Cognitive Radio Networks Under ON/OFF and Poisson Primary Arrivals. IEEE International Symposium on Personal, Indoor and Mobile Radio Communications. 3302-3307.

Chun-ting Chou, Sai Shankar N., Hyoil Kim, and Kang G. Shin. (2007). What and how much to gain by spectrum agility? IEEE Journal on Selected Areas in Communications. 25(3), 576588.

Cruz-Pérez F.A., Serrano-Chávez J., Castellanos-López S.L., and Hernandez-Valdez G. (2017). Approximating Log-Normally Distributed Secondary Service Time by HyperExponential Distribution for the Analytical Performance Evaluation of Cognitive Radio Networks. IEEE International Symposium on Wireless Personal Multimedia Communications, 641-644. 
Fayaz Akhtar, Mubashir Husain Rehmani, Martin Reisslein. (2016). White space: Definitional perspectives and their role in exploiting spectrum opportunities. ELSEVIER, Telecommunication Policy. 40, 319-331.

Filin S., Harada H., Murakami H. and Ishizu K. (2011). International Standarization of Cognitive Radio Systems. IEEE Communications Magazine, 82-89.

García, H. M. Espacios y estructuras desplegables. El fenómeno del pliegue en el diseño contemporáneo. Contexto y Diseño, 141.

Martinez-Bauset J., V Pla., Vidal J.R., Guijarro L., (2013). Approximate Analysis of Cognitive Radio Systems Using Time-Scale Separation and its Accuracy. IEEE Commun. Letters. 17(1), 35-38.

Olsson M. (1998). The EMpht-programme. https://pdfs.semanticscholar.org/fbe5/beb38463 7f861f5d392ce347b7468a197d47.pdf

Pak Kay Tang, Yong Huat Chew, Ling Chuen Ong. (2008). On the Time Distribution of White Space Access Opportunities. IEEE Wireless Communications and Networking Conference. 729-734.

Saleem Y. and Rehmani M.-H. (2014). Primary radio user activity models for cognitive radio networks: A survey. ELSEVIER Journal of Network and Computer Applications, 43, 1-16.

Shanidul H. and Wasim A. (2017). Performance analysis of cognitive radio networks with generalized call holding time distribution of secondary users. Telecommunication Systems, 66 (1), 95-108. 